\title{
Ultraviolet sensitivity of the cosmological sequester
}

\author{
Michaela G. Lawrence $\odot^{*}$ and David Seery $\odot^{\dagger}$ \\ Astronomy Centre, Department of Physics and Astronomy, University of Sussex, \\ Brighton BN1 9QH, United Kingdom
}

(Received 21 July 2020; accepted 16 February 2021; published 11 March 2021)

\begin{abstract}
We revisit the "sequester" proposal of Kaloper, Padilla and collaborators, in which the amplitude of the cosmological constant is decoupled from large contributions due to loops containing Standard Model particles. We review the different formulations of the model that have appeared in the literature, and estimate the importance of a particular class of quantum corrections- those that dress the interaction between the "rigid" scalars and infrared properties of the spacetime such as its 4-volume and integrated curvature. In formulations that do not adequately sequester graviton loops we argue that dressing of these interactions causes further failures of complete sequestration. We estimate the size of the effect and find that it is typically smaller than the cosmological term directly induced by loops containing a single virtual graviton. Meanwhile, in the most developed formulation of the scenario (where a rigid scalar couples to the Gauss-Bonnet density), this dressing can be absorbed into a rescaling of the rigid fields and is therefore harmless.
\end{abstract}

DOI: 10.1103/PhysRevD.103.064018

\section{INTRODUCTION}

It is nearly 40 years since the cosmological constant problem was first stated clearly [1,2]. (For the earlier history, see Ref. [3,4].) Despite immense efforts over the intervening decades, it remains the most enigmatic component of the concordance cosmological model. The problem is simple to state. Observation requires the cosmological constant $\Lambda$ to dominate the present Hubble rate, and therefore $3 H_{0}^{2} M_{\mathrm{P}}^{2} \approx \Lambda$. The measured value of $H_{0}$ gives an estimate $\Lambda \approx 10^{-12} \mathrm{eV}^{4}$. Meanwhile quantum contributions to $\Lambda$ from Standard Model particles are much larger. Why, then, is the measured value so small?

The case for "fine tuning".- - The operational meaning of $\Lambda$ is less clear than other quantities that are known to receive large quantum corrections, such as the running couplings that appear in scattering amplitudes, because it couples only at wave number zero where scattering does not occur. Nevertheless, like any low-energy constant, $\Lambda$ presumably can be divided into an incalculable ultraviolet contribution $\Lambda_{\mathrm{UV}}$ from unknown physics lying above the Standard Model, and an infrared contribution $\Lambda_{\text {IR }}$ generated by quantum corrections with Standard Model particles

\footnotetext{
M.G.Lawrence@sussex.ac.uk

D.Seery@sussex.ac.uk
}

Published by the American Physical Society under the terms of the Creative Commons Attribution 4.0 International license. Further distribution of this work must maintain attribution to the author(s) and the published article's title, journal citation, and DOI. Funded by SCOAP ${ }^{3}$. running in the loops. We expect $\Lambda_{\mathrm{IR}} \sim m_{\mathrm{t}}^{4}$ from loop diagrams containing the top quark, which is the heaviest Standard Model particle. ${ }^{1}$

It follows that $\Lambda=\Lambda_{\mathrm{UV}}+\Lambda_{\mathrm{IR}}$ should be of order $m_{\mathrm{t}}^{4} \sim$ $(175 \mathrm{GeV})^{4}$ or larger unless $\Lambda_{\mathrm{UV}}$ is accurately balanced to cancel large contributions from $\Lambda_{\mathrm{IR}}$. The measurement $\Lambda \sim$ $10^{-12} \mathrm{eV}^{4}$ apparently implies that $\Lambda_{\mathrm{UV}}$ is balanced so that cancellation occurs to roughly 56 decimal places. ${ }^{2}$ The scales that contribute to $\Lambda_{\mathrm{UV}}$ and $\Lambda_{\mathrm{IR}}$ are very different, so there is no reason why $\Lambda_{\mathrm{UV}}$ should be related to Standard Model energies. This makes it unlikely that cancellation happens by accident.

\footnotetext{
${ }^{1}$ It is often said that the low-energy calculation yields $\Lambda_{\mathrm{IR}} \sim M_{\mathrm{P}}^{4}$, but this is not justified. Although the vacuum energy computed with a hard momentum cutoff is quartically divergent, it must be remembered that cutoffs do not track dependence on heavy masses [5]. The low-energy theory cannot yield a trustworthy dependence on any mass scale heavier than it contains itself, and the heaviest mass described by the effective Lagrangian for the Standard Model is the top mass $m_{\mathrm{t}}$. See also Sec. 4.2 of Ref. [6], and Refs. [7-9], which show explicitly that the quartically divergent terms cannot be interpreted as a dark energy component.

If there is a contribution to the cosmological constant of order $\sim M_{\mathrm{P}}^{4}$, it comes from $\Lambda_{\mathrm{UV}}$ and not $\Lambda_{\mathrm{IR}}$. For example, this might happen if local field theory remains valid all the way up to the Planck scale, and the low-energy gravitational force is generated by integrating out one or more particles of mass $\sim M_{\mathrm{P}}$. But the outcome could be different if local field theory fails as a good approximation to Nature at some much lower scale.

The large number of decimal places required is because cancellation has to occur in $\Lambda_{\mathrm{UV}}+\Lambda_{\mathrm{IR}}$.
} 
Unless new physics changes the relationship between $\Lambda$, $\Lambda_{\mathrm{IR}}$ and $\Lambda_{\mathrm{UV}}$, the most plausible conclusion is that whatever determines $\Lambda_{\mathrm{UV}}$ must be constrained by some principle forcing $\Lambda$ to be nearly zero. Such a principle would strongly violate decoupling, because it would make the Wilson coefficients of the low-energy action into highly sensitive functions of the ultraviolet boundary condition. The apparent tuning we observe would be a consequence of this exquisite sensitivity.

It is certainly possible that the correct resolution of the cosmological constant problem involves a failure of decoupling along these lines. Unfortunately, modern ideas in particle physics have not yielded any candidate principle that could be responsible for the smallness of $\Lambda$. Moreover, the failure of decoupling makes robust low-energy model building difficult. For these reasons it is now more common to look for an alternative resolution.

Overview of this paper.-In this paper we revisit the "sequester" proposal of Kaloper \& Padilla [10-13]. This is a concrete scenario for new physics that changes the argument given above by removing ("sequestering") the low-energy contribution from all Standard Model particles. The outcome is that the observed cosmological constant $\Lambda$ would be set by $\Lambda_{\mathrm{UV}}$, unless there are further contributions from new unsequestered sectors.

By itself the sequester (or at least its simplest versions) would not explain the observed magnitude of $\Lambda .{ }^{3}$ Even if all matter species participate in sequestration, there would still be a puzzle if we expect $\Lambda_{\mathrm{UV}}$ to receive contributions larger than $10^{-3} \mathrm{eV}$. This might be the case, for example, if lowenergy Einstein gravity is an effective description generated by integrating out one or more Planck-mass particles. The advantage of the sequester is that the small observed value no longer requires cancellations between $\Lambda_{\mathrm{UV}}$ and $\Lambda_{\mathrm{IR}}$. We express this by saying that its value is technically natural within the Standard Model. Whether or not it is technically natural with respect to the ultraviolet model is a question that can be resolved only when that theory is specified.

The status of arguments based on technical naturalness has been called into question following the discovery of a Higgs particle at $M \sim 125 \mathrm{GeV}$ without new accompanying particles $[14,15]$. In the formulation we are using, "naturalness" has a clear meaning in terms of sensitivityor lack of it - to large corrections between widely separated scales $[16-18] .{ }^{4}$ This is not merely an aesthetic choice, and

\footnotetext{
${ }^{3}$ In Sec. III D we will see that the most developed version of the sequester would absorb $\Lambda_{\mathrm{UV}}$ in addition to $\Lambda_{\mathrm{IR}}$, at the cost of introducing a new cosmological-like term associated with an unknown scale $\mu$. See Eqs. (33) and (34). Therefore, no matter what strategy we choose, it seems that one cannot arrive at an unambiguous prediction for the observed value of $\Lambda$.

${ }^{4}$ This is a broader definition than the original concept of technical naturalness due to t'Hooft [19]. t'Hooft's criterion that a small parameter $y$ is natural if the symmetry of the theory is enlarged in the limit $y \rightarrow 0$ is a sufficient but not necessary condition for widely separated scales to decouple in this sense.
}

accordingly Nature may or may not be "natural" in our sense. Nevertheless, it is reasonable to expect this concept of naturalness to be a useful guide because experience has shown that the vast majority of physical phenomena do decouple in this way.

Clearly we should not be satisfied with a theory in which $\Lambda$ is made technically natural at the expense of other lowenergy constants that receive large ultraviolet corrections. If this occurs we have not removed the problem, but merely translated it from one low-energy sector to another. In this paper we aim to apply this test to the sequester model.

Synopsis.-Two principal variants of the sequester have been discussed in the literature. In the first version, one works in the Einstein frame and couples the sequestered sectors to a conformally rescaled metric. This version was introduced in Refs. [10,11]; see Ref. [20] for a pedagogical description. We describe it as the "Einstein frame model." The conformal rescaling dynamically adjusts mass scales in the sequestered sector relative to the fixed Planck scale. A global constraint couples the cosmological term to this conformal factor, allowing it to absorb contributions from pure matter loops. In this version, loops involving virtual gravitons are known to reintroduce unsequestered corrections to the observed $\Lambda$. We discuss this model and the degree to which it ameliorates ultraviolet sensitivity of the cosmological constant in Sec. II.

A second variant was introduced in Refs. [12,13]. In this version one works in the Jordan frame and there is no auxiliary rescaled metric. There are two global constraints, the first of which couples the gravitational scale to the mean Ricci curvature of spacetime. The second couples the cosmological constant to the total spacetime volume and a physical mass scale $\mu$, which is a priori unknown. The is the "Jordan frame model." In this version one can adjust the way in which the global constraints couple to spacetime curvature so that loops of virtual gravitons are also absorbed. This version of the sequester and its ultraviolet properties are discussed in Sec. III. We conclude in Sec. IV.

Notation.-We work in units where $c=\hbar=1$. The (reduced) Planck mass is $M_{\mathrm{P}} \equiv(8 \pi G)^{-1 / 2}=2.435 \times$ $10^{18} \mathrm{GeV}$. We express the cosmological constant in terms of an energy scale $\Lambda$ with engineering dimension $[\mathrm{M}]^{4}$. The corresponding "cosmological term" in the Einstein equations is $\Lambda$. We generally frame our calculations in Minkowski space to avoid unneeded complexities associated with curved spacetime; because ultraviolet properties do not depend on these curvature scales, this procedure does not forfeit any essential generality.

\section{EINSTEIN FRAME MODEL}

\section{A. The sequester action}

In this section we briefly review the sequester mechanism in Einstein frame [10,11], and discuss its ultraviolet sensitivity. The gravitational action is written in terms of the Einstein-frame metric $g_{\mu \nu}$. Sequestration of one or more 
matter sectors is achieved by coupling them to a conformally rescaled (Jordan-frame) metric $\tilde{g}_{\mu \nu}=\lambda^{2} g_{\mu \nu}$, viz.

$$
\begin{aligned}
S= & \int \mathrm{d}^{4} x \sqrt{-g}\left(\frac{M_{\mathrm{P}}^{2}}{2} R(g)-\Lambda+\Lambda_{\mathrm{UV}}-\lambda^{4} \mathcal{L}_{\mathrm{m}}\left(\tilde{g}^{\mu \nu}, \Psi\right)\right) \\
& +\sigma\left(\frac{\Lambda}{\lambda^{4} \mu^{4}}\right) .
\end{aligned}
$$

If multiple sectors are to be sequestered their actions should appear additively. In Eq. (1), $R(g)=g^{\mu \nu} R_{\mu \nu}(g)$ is the Ricci scalar constructed using the Einstein-frame metric $g_{\mu \nu}, \mathcal{L}_{\mathrm{m}}$ is a matter Lagrangian density, and $\Psi$ schematically stands for the different species of sequestered matter fields. We assume these to be the Standard Model fields. The low energy contribution to the cosmological constant, $\Lambda_{\mathrm{IR}}$, does not appear in Eq. (1) explicitly. It is generated by the infrared part of loop corrections to $\mathcal{L}_{\mathrm{m}}$. The bare cosmological constant (if there is one), plus any contributions from unsequestered sectors that have been integrated out to produce (1), are included in $\Lambda_{\mathrm{UV}}$.

The quantity $\Lambda$ is no longer the combination $\Lambda_{\mathrm{UV}}+\Lambda_{\mathrm{IR}}$, but is rather a new field that can loosely be regarded as a counterterm for $\Lambda_{\mathrm{IR}}$. In particular, although it participates in the path integral, we take $\Lambda$ to have no local degrees of freedom. It is determined classically by extremization of the action. The dimensionless conformal rescaling $\lambda$ is taken to be a field of the same kind.

The global term $\sigma$ is a function of $\Lambda$ and $\lambda$ in the specific dimensionless combination $\Lambda /(\lambda \mu)^{4}$. Critically, it does not couple to either the Einstein- or Jordan-fame metric, and therefore does not source the global gravitational field. The scale $\mu$ has dimension [M], but its precise meaning depends on the definition of $\sigma$. We will discuss its significance in more detail in Sec. III B below. Finally, for reasons to be explained below, we should take $\sigma$ to be an odd function of its argument. It is otherwise assumed to be an arbitrary smooth function.

The rigidity of $\Lambda$ and $\lambda$ is unusual, but can be given a local, microscopic basis in terms of integrals of a four-form flux $F_{4}$ over spacetime [12]. Such a flux is a top-order form in $d=4$ dimensions and therefore acts as a volume form in the integral $\int F_{4}$. In particular, this integral can be written without requiring a metric. Borrowing terminology from thermodynamics, we describe terms such as $\int F_{4}$ that do not scale with $g_{\mu \nu}$ as intensive. Ordinary contributions to the action such as $\int(\star 1)$ are conversely extensive. Notice that if $\sigma$ does not scale at least with the coordinate volume of spacetime, this violates Hawking's suggestion that the action should be additive over cobordant regions in order for quantum gravitational amplitudes to superpose correctly [21].

\section{B. Low-energy phenomenology}

We now consider low-energy solutions to (1). First, notice that the matter contribution in (1) can be written ${ }^{5}$

$$
S_{\mathrm{m}} \equiv-\int \mathrm{d}^{4} x \sqrt{-\tilde{g}} \mathcal{L}_{\mathrm{m}}\left(\tilde{g}^{\mu \nu}, \Psi\right)
$$

Therefore it is clear that the matter fields $\Psi$ are minimally coupled to the Jordan-frame metric $\tilde{g}_{\mu \nu}$. By taking $\tilde{g}_{\mu \nu}$ to be flat up to corrections from the Newtonian potential, it follows that predictions for laboratory measurements in a weak gravitational field will match those of the unsequestered Standard Model.

We conclude that the masses and other properties of the Standard Model reported by the Particle Data Group [22] are those measured in $\tilde{g}_{\mu \nu}$. We denote these experimental scales with a tilde, viz. $\tilde{M}_{Z}, \tilde{m}_{\mathrm{t}}$. They are related to scales measured in the metric $g_{\mu \nu}$ by a conformal rescaling $\tilde{M}_{Z} \rightarrow M_{Z}=\lambda \tilde{M}_{Z}$.

Sequestering low-energy loops.-Extremization of (1) with respect to $\Lambda$ and $\lambda$ yields,

$$
\begin{aligned}
\frac{\sigma^{\prime}}{(\lambda \mu)^{4}} & =\int \mathrm{d}^{4} x \sqrt{-g}, \\
4 \frac{\Lambda}{(\lambda \mu)^{4}} \sigma^{\prime} & =\int \mathrm{d}^{4} x \sqrt{-\tilde{g}} \tilde{T}_{\mu}^{\mu}=\int \mathrm{d}^{4} x \sqrt{-g} T_{\mu}^{\mu},
\end{aligned}
$$

where a prime ' denotes differentiation of $\sigma$ with respect to its argument, and the Jordan-frame energy-momentum tensor $\tilde{T}_{\mu \nu}$ measured with respect to $\tilde{g}^{\mu \nu}$ is defined by

$$
\tilde{T}_{\mu \nu} \equiv-\frac{2}{\sqrt{-\tilde{g}}} \frac{\delta S_{\mathrm{m}}}{\delta \tilde{g}^{\mu \nu}}
$$

A similar definition applies for the Einstein-frame energymomentum tensor $T_{\mu \nu}$, which is measured with respect to $g^{\mu \nu}$. The two definitions are related by $\tilde{T}_{\mu \nu}=\lambda^{-2} T_{\mu \nu}$. We assume $\sigma^{\prime} \neq 0$ at the extremum. To allow consistent solutions with $\Lambda<0$ but $\lambda>0$ we require $\sigma^{\prime}(x)$ to be even, and hence $\sigma(x)$ must be odd, as stated above.

Taking the ratio of Eqs. (3b) and (3a) yields a constraint for $\Lambda$,

$$
\Lambda=\frac{1}{4}\left\langle\left\langle T_{\mu}^{\mu}\right\rangle\right\rangle
$$

where $\langle\langle\cdots\rangle\rangle$ denotes spacetime averaging in the metric $g_{\mu \nu}$, i.e., $\left\langle\langle Q\rangle \equiv \int \mathrm{d}^{4} x \sqrt{-g} Q / \int \mathrm{d}^{4} x \sqrt{-g}\right.$. Since we assume $\sigma$ is differentiable, Eq. (3a) requires the volume of spacetime to be finite if we wish to avoid $\lambda=0$. (This would conformally rescale all masses in the sequestered

\footnotetext{
${ }^{5}$ We take this as the definition of the matter action $S_{\mathrm{m}}$.
} 
sector to zero.) It follows that the spacetime average $\langle\langle Q\rangle$ can be defined, even if it is difficult to evaluate in practice.

The Einstein equation that follows from (1) is

$$
\begin{aligned}
M_{\mathrm{P}}^{2} G_{\mu \nu} & =T_{\mu \nu}-\left(\Lambda-\Lambda_{\mathrm{UV}}\right) g_{\mu \nu} \\
& =T_{\mu \nu}-\frac{1}{4}\left\langle\left\langle T^{\mu}{ }_{\mu}\right\rangle\right\rangle g_{\mu \nu}+\Lambda_{\mathrm{UV}} g_{\mu \nu},
\end{aligned}
$$

where $G_{\mu \nu}(g)=R_{\mu \nu}(g)-R(g) g_{\mu \nu} / 2$ is the usual Einstein tensor constructed from $g_{\mu \nu}$. As explained above, the $\sigma$ term in the action does not couple to $g_{\mu \nu}$ and therefore does not source a long-wavelength gravitational field. Diffeomorphism invariance guarantees that any matter loops renormalize the cosmological term in $\mathcal{L}_{\mathrm{m}}$ measured using $\tilde{g}_{\mu \nu}$ (see Fig. 2), and therefore

$$
\tilde{T}_{\mu \nu}=\tilde{\Lambda}_{\mathrm{IR}} \tilde{g}_{\mu \nu}+\tilde{\tau}_{\mu \nu}(\tilde{g}, \Psi, \cdots),
$$

where the "subtracted" energy-momentum tensor $\tilde{\tau}_{\mu \nu}(\tilde{g}, \Psi, \ldots)$ vanishes outside matter. We have added a tilde to $\Lambda_{\mathrm{IR}}$ to indicate that it is built from scales such as $\tilde{m}_{\mathrm{t}}$ measured in a homogeneous gravitational field. When expressed in terms of $T_{\mu \nu}$ we obtain

$$
T_{\mu \nu}=\lambda^{4} \tilde{\Lambda}_{\mathrm{IR}} g_{\mu \nu}+\lambda^{2} \tilde{\tau}_{\mu \nu}(\tilde{g}, \Psi, \ldots),
$$

It follows that the effective Einstein equation can be written

$$
M_{\mathrm{P}}^{2} G_{\mu \nu}=\tau_{\mu \nu}-\frac{1}{4}\left\langle\left\langle\tau_{\rho}^{\rho}{ }_{\rho}\right\rangle g_{\mu \nu}+\Lambda_{\mathrm{UV}} g_{\mu \nu} .\right.
$$

The conclusion is that, in the Einstein equation, the lowenergy contribution $\Lambda_{\mathrm{IR}}$ is removed to all orders in the loop expansion of $\mathcal{L}_{\mathrm{m}}$.

What has been achieved?-To reiterate, this does not "solve" the cosmological constant problem because we still have no means to estimate $\Lambda_{\mathrm{UV}}$. Depending on the ultraviolet model, it may be large. But since an estimate of $\Lambda_{\mathrm{UV}}$ was never the aim of the sequester, this criticism is unfair. Instead, what has been achieved is that if $\Lambda_{\mathrm{UV}}$ can somehow be made small, its impact on the global spacetime geometry is not destabilized by loops at much lower scales.

Loosely speaking, this analysis shows that the sequester is not a field theory mechanism, in the sense that the properties of loops are unmodified in the ultraviolet. Rather, we have added a new form of matter $\Lambda$ that is constrained by its field equation to cancel the portion of the vacuum energy sourced by matter loops. Ordinarily this would be of no benefit, because the energy density associated with $\Lambda$ would itself gravitate. As explained above, the special feature of the action for $\Lambda$ is that its $\sigma$ part does not source any gravitational field. Heuristically, this allows us to "degravitate" or "sequester" the vacuum energy by storing it in $\sigma$. When stored in this way the matter loops are gravitationally inert.
After vacuum loops have been sequestered, the effective source term for the gravitational field is the subtracted energy-momentum tensor $\tau_{\mu \nu}$ computed in the metric $g_{\mu \nu}$, together with a correction from its spacetime volume average $\left\langle\left\langle\tau^{\rho}{ }_{\rho}\right\rangle\right\rangle$. The size of this correction was estimated in Refs. [10,11], who considered a model in which the unsequestered contribution $\Lambda_{\mathrm{UV}}$ was set to zero.

Nevertheless, there is something surprising about this outcome. We are still working in the context of local field theory, with its characteristic poor control of ultraviolet effects. Where has the original ultraviolet sensitivity of the cosmological term gone? The sequester does contain a new physical ingredient, in the form of the $\sigma$-term that is shielded from gravity. However, we have not introduced a new physical principle that forces $\Lambda$ to capture the entirety of $\Lambda_{\mathrm{IR}}$ in this non-gravitating sector. Therefore one might worry that quantum corrections "detune" the dynamical equation for $\Lambda$, preventing complete sequestration of $\Lambda_{\mathrm{IR}}$ and reintroducing the low-energy cosmological term.

Radiative corrections.-Indeed, when discussing any proposed solution to the cosmological constant problem it is never sufficient to work at tree level. Like any naturalness problem, the cosmological constant problem is intrinsically quantum mechanical because it is only in a quantum theory that loop corrections generate direct correlations between widely separated scales. The conclusion is that radiative corrections must be included before we can judge the merits of any particular proposal.

A subset of relevant corrections were considered in Refs. [10-13]. First, these authors considered a symmetry $\lambda \rightarrow \Omega \lambda, g_{\mu \nu} \rightarrow \Omega^{-2} g_{\mu \nu}, \Lambda \rightarrow \Omega^{4} \Lambda$ under which Eq. (1) is invariant. They argued this was sufficient to guarantee that, to all orders in matter loops, $\Lambda_{\mathrm{IR}}$ would couple to Eq. (3b) like the tree-level vacuum energy. In our presentation this symmetry is implied by coupling $\mathcal{L}_{\mathrm{m}}$ to the Jordan-frame metric $\tilde{g}_{\mu \nu}$ but $\Lambda$ to the Einstein-frame metric $g_{\mu \nu}$. The looplevel behavior of $\Lambda_{\mathrm{IR}}$ then follows from diffeomorphism invariance with respect to $\tilde{g}_{\mu \nu}$. We will give a pedestrian proof of these properties in Sec. IIC below, based on analysis of Feynman diagrams. As we show there, like all global symmetries, this one is broken by coupling to gravity.

Second, Refs. [10-13] studied the symmetry $\Lambda \rightarrow \Lambda+\lambda^{4} \nu^{4}, \quad \mathcal{L}_{\mathrm{m}} \rightarrow \mathcal{L}_{\mathrm{m}}-\nu^{4}$ which they suggested would guarantee that $\Lambda$ absorbed $\Lambda_{\mathrm{IR}}$ to all orders in matter loops. [That is, that $\Lambda$ and $T_{\mu \nu}$ would appear additively in the Einstein equation as in Eq. (6).] This last symmetry is not in fact a transformation of the fields that participate in the action, and is not respected by quantum corrections.

\section{Ultraviolet sensitivity in Einstein frame}

This list does not exhaust the loop corrections to Eq. (1). In particular, the analysis of Refs. [10-13] leaves open the 
issue of (i) corrections to the intensive global function $\sigma$ that "stores" the unwanted large loop terms; and (ii) corrections to the extensive interaction $\int \mathrm{d}^{4} x \sqrt{-g} \Lambda$. To study corrections to $\sigma$ would require a microscopic theory that explains how the flux $F_{4}$ is supported. The sequester proposal does not aim to provide such a description. (For recent attempts to describe an ultraviolet completion of this kind, see Refs. [23-27].) We comment on this in Sec. IV. On the other hand, the interaction term couples to spin-2 excitations of the metric $g_{\mu \nu}$, and will therefore be "dressed" by loops containing off-shell quanta associated with these excitations (cf. Ref. [28]). This is a model independent effect, in the sense that it does not depend on the microscopic origin of $F_{4}$. In this section we aim to enumerate these corrections and quantify their impact.

Loops respect diffeomorphism invariance.-First, we pause to prove the property stated above, that pure matter loops generate a cosmological term scaling as $\lambda^{4}$ to all orders in the loop expansion. This follows from diffeomorphism invariance with respect to $\tilde{g}_{\mu \nu}$, but can also be proved by direct analysis of Feynman diagrams. The results will assist us in an analysis of corrections to the $\Lambda$ coupling, to be given below.

Consider any operator in $\mathcal{L}_{\mathrm{m}}$ formed from a monomial of $n_{b}$ bosonic fields and $n_{f}$ fermionic fields. After replacing measured mass scales $\tilde{M}$ by their conformally rescaled equivalents $M=\lambda \tilde{M}$, and performing the same replacement $k=\lambda \tilde{k}$ for momenta, it can be checked that such an operator scales like $\lambda^{n_{b}+3 n_{f} / 2}$. Meanwhile, a boson propagator scales like $\lambda^{-2}$ whereas a fermion propagator scales like $\lambda^{-3}$. Therefore a diagram containing $I_{b}$ internal boson lines, $E_{b}$ external boson lines, $I_{f}$ internal fermion lines, and $E_{f}$ external fermion lines will scale like $\lambda^{D}$, where

$D=-2 I_{b}-2 E_{b}-3 I_{f}-3 E_{f}+\sum_{i} N_{i}\left(n_{b, i}+\frac{3}{2} n_{f, i}\right)$.

$N_{i}$ is the number of vertices of type $i$, each of which contains $n_{b, i}$ bosonic fields and $n_{f, i}$ fermionic fields.

Each diagram must satisfy the topological identity $2 I+E=\sum_{k} N_{k} n_{k}$, where now $I$ denotes the total number of internal lines (whether bosons or fermions), $E$ denotes the total number of external lines, $N_{k}$ denotes the number of vertices of type $k$, and each type- $k$ vertex connects $n_{k}$ lines. To translate to an operator in the effective action we should amputate external lines. Applying the identity separately to the bosonic and fermionic components of the amputated diagram, it follows that the effective operator will scale like $\lambda^{D_{\text {amp }} \text {, where }}$

$$
D_{\text {amp }}=E_{b}+\frac{3}{2} E_{f}
$$

(This analysis applies even if the bosonic and fermionic components are disconnected, provided the assignment of internal and external lines is the one appropriate for the entire diagram.) No matter how complex the diagram, Eq. (11) involves only the total number of amputated bosonic and fermionic lines. Such a diagram will renormalize operators that are polynomial in $E_{b}$ bosonic fields and $E_{f}$ fermionic fields. The $\lambda$-dependence of this renormalization will be $\lambda^{E_{b}+3 E_{f} / 2}$, the same as we deduced above for unrenormalized operators in $\mathcal{L}_{\mathrm{m}}$. The conclusion, as has already been stated, is that pure matter loops preserve the $\lambda$-dependence of the coupling in Eq. (1). ${ }^{6}$

Stability of global constraint.-Next, we argue that detuning the dynamical equation for $\Lambda$ can prevent complete sequestration. Specifically, to obtain complete cancellation in the Einstein equation, the factor of 4 that appears on the far left of Eq. (3b) is required to match a factor of 4 from the trace of the metric in $T^{\mu}{ }_{\mu}$. Even a small mismatch of these factors will leave a residual low-energy cosmological term in Eq. (6).

While the 4 from the trace $\delta^{\mu}{ }_{\mu}$ cannot be modified by ultraviolet effects, the other factor of 4 is a consequence of the power $\lambda^{-4}$ appearing in the combination $\Lambda /(\lambda \mu)^{4}$ that enters the global function $\sigma$. We will argue below that this factor can be renormalized by ultraviolet effects. It follows that Eq. (1) may receive significant corrections from high energies, and therefore fails the test for naturalness in the sense we have defined.

How sensitive is the successful operation of the sequester to the precise factor 4 in Eq. (3b)? If it is replaced by $4(1+\alpha)$, the analogue of the sequestered Einstein equation (9) becomes

$$
\begin{aligned}
M_{\mathrm{P}}^{2} G_{\mu \nu}= & \frac{\alpha}{1+\alpha} \lambda^{4} \tilde{\Lambda}_{\mathrm{IR}} g_{\mu \nu}+\tau_{\mu \nu} \\
& -\frac{1}{4(1+\alpha)}\left\langle\left\langle\tau_{\mu}^{\mu}\right\rangle\right\rangle g_{\mu \nu}+\Lambda_{\mathrm{UV}} g_{\mu \nu} .
\end{aligned}
$$

As expected, there is now incomplete cancellation of $\Lambda_{\mathrm{IR}}$. To estimate the magnitude of the residual cosmological term requires a numerical estimate for $\lambda$. In a finite universe, Eq. (3a) yields

$$
\lambda \sim \sigma^{\prime} \frac{H_{\text {age }}}{\mu} .
$$

The mass scale $H_{\text {age }}$ was introduced in Ref. [11] and specifies the lifetime of the universe. This roughly determines the spacetime volume,

\footnotetext{
${ }^{6}$ Recall that this scaling applies after conformal redefinition of the masses. From inspection of Eq. (1), one might expect the cosmological term generated by matter to scale as $\lambda^{4} \tilde{M}_{\mathrm{SM}}^{4}$, where $\tilde{M}_{\mathrm{SM}}$ is some characteristic Standard Model scale. This does not conflict with (11) for $E_{b}=E_{f}=0$ because after rescaling $M_{\mathrm{SM}}=\lambda \tilde{M}_{\mathrm{SM}}$ the cosmological term scales as $\lambda^{0}$ as claimed.
} 


$$
\frac{1}{H_{\text {age }}^{4}} \sim \int \mathrm{d}^{4} x \sqrt{-g}
$$

Clearly $H_{\text {age }}<H_{0} \sim 10^{-33} \mathrm{eV}$.

Reference [11] suggested that $\sigma$ should be engineered to obtain $\lambda=O(1)$. In this case, the effective gravitating cosmological constant is roughly $\alpha \Lambda_{\mathrm{IR}} \sim \alpha \tilde{\Lambda}_{\mathrm{IR}} \sim \alpha \tilde{m}_{\mathrm{t}}^{4}$, assuming $|\alpha| \ll 1$. With these estimates, $|\alpha|$ must inherit the tuning to 56 decimal places that was previously required for the combination $\Lambda_{\mathrm{UV}}+\Lambda_{\mathrm{IR}}$. If $\lambda$ is made smaller then $\alpha$ can be relaxed accordingly, but this scenario encounters other difficulties [11].

Extensive corrections to the $\Lambda$ coupling. - Let us now estimate the model-independent corrections to the extensive coupling $-\Lambda V$, where $V=\int \mathrm{d}^{4} x \sqrt{-g}$.

First, consider the two-loop correction that appears in the left-hand diagram of Fig. 1. Regarded as a contribution to the quantum effective action, this contains a single insertion of a $\Lambda$ vertex which is "bridged" to a pure Standard Model loop by a pair of spin-2 excitations. This diagram is part of a larger class of diagrams, represented by the right-hand part of Fig. 1, in which an arbitrary number of $\Lambda$ insertions are bridged to a Standard Model subdiagram (of arbitrary complexity) by graviton lines.

(These diagrams are not the only sources of renormalization for the $\Lambda$ coupling. We could equally well consider diagrams in which the $\Lambda$ insertions are embedded within the Standard Model subdiagram. For our purpose, it suffices to consider only a subclass of possible renormalizations.)

According to the analysis given above, the $\lambda$ dependence of this Standard Model subdiagram can be computed from Eq. (10). This time we are not amputating external lines, so the scaling is $\lambda^{D_{\text {sub }}}$ where $D_{\text {sub }}=-E_{b}-3 E_{f} / 2$. Because the subdiagram connects to the ring of $\Lambda$ insertions via
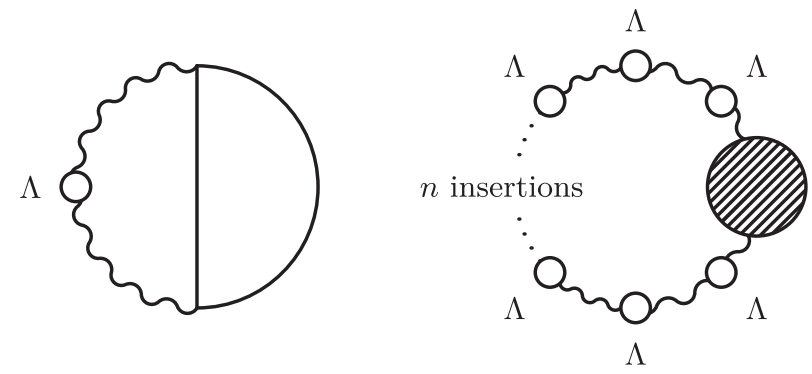

FIG. 1. Loops renormalizing the coupling of $\Lambda$ to the spacetime volume. Left: loop with single insertion of $\Lambda$ vertex, represented by the open circle. Wiggly lines represent spin-2 excitations of the metric $g_{\mu \nu}$; solid lines represent Standard Model fields. This diagram renormalizes the coefficient of $\Lambda$ in $\sigma$. Right: loop containing $n$ insertions of $\Lambda$. This diagram renormalizes the coefficient of $\Lambda^{n}$ in $\sigma$. The shaded circle represents any Standard Model sub-diagram. The left-hand diagram is a particularly simple example of the class represented by the right-hand diagram. graviton lines (which do not scale with $\lambda$ ) we have $E_{b}=E_{f}=0$. Meanwhile, counting the number of $\Lambda$ insertions and a factor $M_{\mathrm{P}}^{-2}$ for each graviton propagator, we conclude that such a diagram produces an operator $\mathcal{O}$ in the quantum effective action of the form

$$
\begin{aligned}
\mathcal{O}_{n} & =\frac{c_{n}}{(2 \pi)^{4(L+1)}} M_{\mathrm{SM}}^{4} \frac{\Lambda}{M_{\mathrm{P}}^{4}}\left(\frac{\Lambda}{M_{\mathrm{P}}^{2} M_{\mathrm{SM}}^{2}}\right)^{n-1} \\
& =\frac{c_{n}}{(2 \pi)^{4(L+1)}} \Lambda^{n} M_{\mathrm{P}}^{-2(n+1)} M_{\mathrm{SM}}^{6-2 n},
\end{aligned}
$$

where $L$ counts the number of loops in the Standard Model subdiagram and $c_{n}$ is a Wilson coefficient that can be taken to be of order unity. The scale $M_{\mathrm{SM}}$ represents a typical Standard Model mass. After replacing $M_{\mathrm{SM}}$ by its experimentally-measurable counterpart $\tilde{M}_{\mathrm{SM}} \sim \mathrm{TeV}$, it follows that $\mathcal{O}$ scales like $\lambda^{6-2 n}$.

To validate Eq. (15) we have evaluated the explicit twoloop diagram given in the left-hand part of Fig. 1, for which $n=1$. Using dimensional regularization as the ultraviolet regulator, this yields the expected scaling

$$
\mathcal{O}_{1}=\frac{c_{1}}{(2 \pi)^{8}} \frac{\lambda^{4} \tilde{M}_{\mathrm{SM}}^{4}}{M_{\mathrm{P}}^{4}} \Lambda
$$

The factor $(2 \pi)^{-8}$ is included from the measure on the loop integrals. Equation (16) will be the leading correction provided $|\Lambda| \lesssim\left(M_{\mathrm{P}} M_{\mathrm{SM}}\right)^{2}$. This will generally be the case where $\Lambda$ is dynamically constrained to sequester a loop contribution of order $M_{\mathrm{SM}}^{4}$. The field equations corrected by $\mathcal{O}_{1}$ are

$$
\begin{gathered}
\left(1-\lambda^{4} \epsilon\right) \frac{\sigma^{\prime}}{(\lambda \mu)^{4}}=\int \mathrm{d}^{4} x \sqrt{-g}, \\
4\left(1+\lambda^{4} \epsilon\right) \frac{\Lambda}{(\lambda \mu)^{4}} \sigma^{\prime}=\int \mathrm{d}^{4} x \sqrt{-g} T_{\mu}^{\mu}, \\
M_{\mathrm{P}}^{2} G_{\mu \nu}=T_{\mu \nu}-\left(1+\lambda^{4} \epsilon\right) \Lambda g_{\mu \nu}+\Lambda_{\mathrm{UV}} g_{\mu \nu},
\end{gathered}
$$

where $\epsilon \equiv(2 \pi)^{-8} c_{1}\left(\tilde{M}_{\mathrm{SM}} / M_{\mathrm{P}}\right)^{4} \ll 1$ and we have dropped terms of $O\left(\epsilon^{2}\right)$. After eliminating $\Lambda$, the Einstein equation can be written, still up to $O(\epsilon)$ [cf. (12)],

$$
M_{\mathrm{P}}^{2} G_{\mu \nu}=\left(\left(\lambda^{4} \epsilon\right) \Lambda_{\mathrm{IR}}+\Lambda_{\mathrm{UV}}\right) g_{a b}+\tau_{\mu \nu}-\frac{1}{4}\left\langle\left\langle\tau_{\rho}^{\rho}\right\rangle\right\rangle .
$$

We have omitted $O(\epsilon)$ corrections if they merely perturb existing terms of order unity. The conclusion is that $\mathcal{O}_{1}$ corrects Eqs. (17a)-(17b) differently, and therefore renormalizes the relative factor 4 between their left-hand sides.

As in the analysis leading to Eq. (12), this $\mathcal{O}_{1}$-corrected factor no longer cancels the exact 4 coming from $\delta^{\mu}{ }_{\mu}$, leaving a residual loop term in Eq. (18). This outcome is 
practically inevitable. In this formulation of the sequester, one is attempting to balance a protected topological quantity $\delta^{\mu}{ }_{\mu}$ against the properties of a class of unprotected Lagrangian operators. One is immune from ultraviolet effects but the other is not, making the balance extremely delicate.

Size of residual loop-level term. - How significant is this effect? Taking $\lambda$ of order unity and $\tilde{M}_{\mathrm{SM}}$ of order $1 \mathrm{TeV}$ makes $\epsilon$ of order $10^{-68}$, or possibly as large as $10^{-62}$ if we omit the loop-counting factor $(2 \pi)^{-8}$ on the assumption it is partially cancelled by combinatorial factors. Meanwhile if $\tilde{\Lambda}_{\mathrm{IR}}$ is also of order $1 \mathrm{TeV}$ then the residual loop-sourced cosmological term in (18) is of order $\left(10^{-4} \mathrm{eV}\right)^{4}$ to $\left(10^{-5} \mathrm{eV}\right)^{4}$, or $\left(10^{-4} \mathrm{eV}\right)^{4}$ to $\left(10^{-3} \mathrm{eV}\right)^{4}$ if the loopcounting factor is omitted. This is on the boundary of being acceptable given current observational constraints.

The outcome is that whether the Einstein-frame model can survive ultraviolet corrections to the extensive coupling is model-dependent. Assuming the sequestered sector to be the Standard Model gives a barely acceptable phenomenology, with success or failure largely dependent on whether $\lambda$ is larger or smaller than unity.

Alternatively, if the sequestered sector contains particles that are heavier than the Standard Model-for example, perhaps from a higher-lying supersymmetric sector-then the model is unlikely to survive unless $\lambda$ is significantly smaller than unity. If the heaviest sequestered mass scale is even $10 \mathrm{TeV}$ then the residual cosmological constant is already in excess of the observed value.

\section{JORDAN FRAME MODEL}

\section{A. Graviton loop corrections}

The radiative corrections described in Sec. II C above involved loop diagrams containing virtual Einstein-frame gravitons that dress the $\Lambda$ coupling to the spacetime volume. There is a further class of diagrams of this type that are significant in the Einstein frame. These are loop diagrams containing virtual gravitons that contribute to the low-energy cosmological constant. As explained in Ref. [13], and as we will review below, these contributions escape the sequester.

Consider the left-hand diagram of Fig. 2, which is a loop diagram containing only matter fields. As explained in the discussion leading to Eq. (11), this diagram has $E_{b}=E_{f}=$ 0 external lines and therefore scales like $\lambda^{0}$ multiplied by $M_{\mathrm{SM}}^{4}$. Expressed in terms of the mass measured in a homogeneous gravitational field this is $\propto \lambda^{4} \tilde{M}_{\mathrm{SM}}$.

Diagrams containing virtual gravitons.-Now consider the right-hand diagram of Fig. 2. In addition to matter fields (represented by the solid lines), this contains an internal graviton (represented by the wiggly line). Each graviton propagator is proportional to the fixed Planck scale $M_{\mathrm{P}}^{-2}$ with no conformal rescaling. It follows on dimensional grounds that a renormalization of the cosmological term
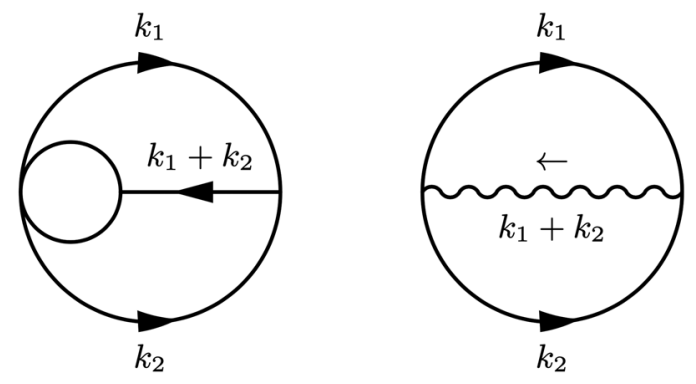

FIG. 2. Diagrams contributing to renormalization of the cosmological constant in the Einstein frame. Solid lines represent generic Standard Model particles, and wiggly lines represent spin-2 excitations of the metric $g_{\mu \nu}$. Left: pure Standard Model loop. This diagram scales like $\lambda^{4}$ when expressed in terms of experimentally-measured mass scales, and is captured by the sequester. Right: mixed Standard Model and graviton loop. Because the Einstein-frame graviton propagator is proportional to the hard scale $M_{\mathrm{P}}^{-2}$, this diagram must scale like $\lambda^{6}$ rather than $\lambda^{4}$. As explained in the main text, this implies it is not captured by the sequester in Einstein frame.

with zero external lines, any number of internal matter lines, and $n_{g}$ internal graviton lines, will scale as $M_{\mathrm{SM}}^{4+2 n_{g}} / M_{\mathrm{P}}^{2 n_{g}} \propto \lambda^{4+2 n_{g}}{ }^{7}$ If $n_{g} \neq 0$ these diagrams do not preserve the $\lambda$ dependence of Eq. (1) [13].

\section{B. Jordan-frame formulation}

To solve this, Ref. [13] proposed an alternative description of the sequester that we review below. It is based on a reformulation of Eq. (1) in the Jordan frame [12]. We wish to analyze the ultraviolet properties of this formulation separately, so we discuss it here before going on to consider the problem of capturing diagrams containing virtual gravitons.

In Eq. (1) the gravitational action is built from $g_{\mu \nu}$, but matter couples to $\tilde{g}_{\mu \nu}$. The conformal factor between $g_{\mu \nu}$ and $\tilde{g}_{\mu \nu}$ adjusts the importance of the matter action $\mathcal{L}_{\mathrm{m}}$ relative to the fixed Einstein term $R(g)$. Alternatively, one can build the action solely from the Jordan-frame metric, leaving the relative importance of the Einstein term as a free parameter,

$$
\begin{aligned}
S= & \int \mathrm{d}^{4} x \sqrt{-g}\left(\frac{\kappa^{2}}{2} R-\Lambda+\Lambda_{\mathrm{UV}}-\mathcal{L}_{\mathrm{m}}\right) \\
& +\sigma\left(\frac{\Lambda}{\mu^{4}}\right)+\hat{\sigma}\left(\frac{\kappa^{2}}{M_{\mathrm{P}}^{2}}\right) .
\end{aligned}
$$

This is the Jordan-frame formulation of the sequester. The gravitational coupling is set by $\kappa$, which is related to $M_{\mathrm{P}}$ by the global term $\hat{\sigma}$. As with $\sigma$, this should be a smooth

\footnotetext{
${ }^{7}$ Such scalings are possibly modified by powers of logarithms, but we drop these unless they are dominant.
} 
function of its argument and is assumed to be produced by integration of a second flux, $\int \hat{F}_{4}$. The Einstein frame metric $g_{\mu \nu}$ does not appear.

Sequestration of low-energy loops.-The field equations that follow from (19) are

$$
\begin{gathered}
\kappa^{2} G_{\mu \nu}=T_{\mu \nu}-\left(\Lambda-\Lambda_{\mathrm{UV}}\right) g_{\mu \nu}, \\
\frac{1}{\mu^{4}} \sigma^{\prime}\left(\frac{\Lambda}{\mu^{4}}\right)=\int \mathrm{d}^{4} x \sqrt{-g}, \\
\frac{1}{M_{\mathrm{P}}^{2}} \hat{\sigma}^{\prime}\left(\frac{\kappa^{2}}{M_{\mathrm{P}}^{2}}\right)=-\int \mathrm{d}^{4} x \sqrt{-g} \frac{R}{2} .
\end{gathered}
$$

Using the definition of spacetime average $\langle\langle\cdots\rangle\rangle$ given in Sec. II B, Eqs. (20b)-(20c) require

$$
\left\langle\langle R\rangle=-2 \frac{\mu^{4}}{M_{\mathrm{P}}^{2}} \frac{\hat{\sigma}^{\prime}}{\sigma^{\prime}} .\right.
$$

Meanwhile, tracing the Einstein equation (20a) and taking the spacetime average, we find

$$
\left.\langle\langle R\rangle\rangle=-\frac{1}{\kappa^{2}}\left[\left\langle T_{\mu}^{\mu}\right\rangle\right\rangle-4\left(\Lambda-\Lambda_{\mathrm{UV}}\right)\right] .
$$

Equations (21) and (22) must hold simultaneously, and therefore

$$
\Lambda-\Lambda_{\mathrm{UV}}=\frac{1}{4}\left\langle\left\langle T_{\mu}^{\mu}\right\rangle\right\rangle-\frac{\mu^{4}}{2} \frac{\kappa^{2}}{M_{\mathrm{P}}^{2}} \frac{\hat{\sigma}^{\prime}}{\sigma^{\prime}}
$$

Finally, we replace $\Lambda$ in the Einstein equation to obtain

$$
\kappa^{2} G_{\mu \nu}=T_{\mu \nu}-\frac{1}{4}\left\langle\left\langle T_{\mu}^{\mu}\right\rangle\right\rangle+\frac{\mu^{4}}{2} \frac{\kappa^{2}}{M_{\mathrm{P}}^{2}} \frac{\hat{\sigma}^{\prime}}{\sigma^{\prime}} g_{\mu \nu} .
$$

Relation between Einstein- and Jordan-frame.-The Einstein- and Jordan-frame formulations are related by a change of frame, and therefore must presumably be regarded as equivalent. This equivalence holds even up to quantum corrections provided one is sufficiently careful to include contributions from the transformation Jacobian; see, e.g., Ref. [29]. The key issue to be addressed is how ultraviolet modes enter each formulation, to be discussed in $\S$ II C.

Before doing so, we enumerate the principal differences between the sequester phenomenology in Einstein frame and Jordan frame. First, in Jordan frame, not only the lowenergy loop contribution $\Lambda_{\mathrm{IR}}$ is sequestered, but also the ultraviolet part $\Lambda_{\mathrm{UV}}$. This happens because both sources for the cosmological term now couple to the Jordan-frame metric. The distinction between them is therefore arbitrary at the level of the Einstein equation. We will see below that this emerges from a more general conclusion, that fluctuations coupling to the Jordan-frame metric (including gravitons) are sequestered, whereas fluctuations coupling to the Einstein-frame metric are not.

Second, the critical factor of $1 / 4$ in the combination $T_{\mu \nu}-\left\langle\left\langle T^{\mu}{ }_{\mu}\right\rangle\right\rangle / 4$ is not ultraviolet sensitive. In particular, it is no longer produced by balancing a topological invariant against the properties of a particular group of Lagrangian operators. Instead, the factor of 1/4 in Eqs. (20b) and (24) is also produced by a trace. Therefore it is not corrected by extensive renormalizations of the coupling of $\Lambda$ to spacetime. We will consider below what is the effect of these renormalizations in the Jordan frame.

Third, the Jordan frame formulation generates a residual cosmological-like term. This is the last term in (24). Assuming $\kappa^{2} \sim M_{\mathrm{P}}^{2}$ and $\sigma^{\prime} \sim \hat{\sigma}^{\prime} \sim O(1)$, it yields a residual cosmological constant of order $\mu^{4}$. Ref. [13] argued that this contribution is at least radiatively stable because it arises from the intensive term $\sigma$, which does not couple either to $g_{a b}$ or the matter fields in $\mathcal{L}_{\mathrm{m}}$. It is therefore uncorrected by matter and graviton loops. On the other hand, depending on its origin, $\sigma$ might be susceptible to other loop corrections associated with unknown mass scales. If so, $\mu$ must apparently be associated with the lowest of these scales, because it is the most relevant terms involving $\Lambda$ that dominate Eq. (22). (However, it should be remembered that $\mu$ does not have a precise meaning until we specify the typical size of Taylor coefficients in $\sigma$.)

This does not preclude the possibility that $\mu$ could typically be large. As with the cosmological constant itself this need not be fatal for the model, because we can always suppose that the renormalized value of $\mu$ is lower than its natural scale. If we choose to do so, however, then presumably we encounter a new naturalness problem in the $\Lambda$ sector. In particular, $\Lambda$ must become relevant at a very low energy scale $\sim\left(10^{-3} \mathrm{eV}\right)^{4}$ to avoid an unwanted large contribution.

At the level of the effective theory (19) there is nothing further that can be said to set our expectations about the typical size of $\mu$. To do so would require a detailed microscopic theory of the fluxes and how they are sourced. Such a theory could be used to compute corrections to the functions $\sigma$ and $\hat{\sigma}$. In this connection, see Refs. [23,25].

\section{Ultraviolet dependence in Jordan frame}

In Eq. (19) there will be extensive renormalizations of the coupling of $\kappa$ and $\Lambda$ to spacetime. Note that $\Lambda$ couples to the spacetime volume, whereas $\kappa^{2}$ couples to the integrated curvature $\int \mathrm{d}^{4} x \sqrt{-g} R$. Renormalizations of the $\Lambda$ coupling were considered above and are unchanged in this theory. Renormalization of the $\kappa$ coupling will arise from diagrams analogous to those of Fig. 1, but with insertions of $\kappa^{2} R$ rather than $\Lambda$. (As before, the class of diagrams shown on the right-hand side of Fig. 1 does not exhaust the contributions at a given order in $\kappa^{2} R$, but they 
provide a representative class that is simple to study.) The leading effects can be summarized by the replacements

$$
\begin{aligned}
\frac{\kappa^{2}}{2} R & \rightarrow \frac{\kappa^{2}}{2}(1+\alpha \epsilon) R, \\
\Lambda & \rightarrow(1+\beta \epsilon) \Lambda
\end{aligned}
$$

where $\alpha$ and $\beta$ are $O(1)$ Wilson coefficients, and $\epsilon$ is defined by

$$
\epsilon \equiv \frac{1}{(2 \pi)^{8}} \frac{M_{\mathrm{SM}}^{4}}{\kappa^{4}} \ll 1 .
$$

To account for renormalizations of the low-energy cosmological constant from diagrams including virtual gravitons, as in the right-hand diagram of Fig. 2, we include a representative term $\gamma M_{\mathrm{SM}}^{6} / \kappa^{2}$ in $\mathcal{L}_{\mathrm{m}}$, where $\gamma$ is another $O(1)$ coefficient. A contribution of this form will be generated by diagrams such as the right-hand side of Fig. 2 containing a single internal graviton line. It would typically be accompanied by contributions of higher order in $\kappa^{-2}$ from diagrams containing two or more internal graviton lines, but if the scale $M_{\mathrm{SM}}$ of the sequestered sector is far below the Planck scale then the one-graviton diagram will be dominant. Notice that this term will contribute to the $\kappa$ field equation. This is the origin of the mismatch that allows such contributions to escape complete sequestration.

After a short calculation, it follows that the effective Einstein equation in this model can be written, up to $O(\epsilon)$,

$$
\begin{aligned}
\kappa^{2} G_{\mu \nu}= & (1-\alpha \epsilon) T_{\mu \nu}-(1-(\alpha+\beta) \epsilon) \frac{\left\langle\left\langle T_{\rho}^{\rho}\right\rangle\right\rangle}{4} g_{\mu \nu} \\
& +\beta \epsilon \Lambda_{\mathrm{UV}} g_{\mu \nu}-\frac{\gamma}{2} \frac{M_{\mathrm{SM}}^{6}}{\kappa^{2}}+\frac{\mu^{4}}{2} \frac{\kappa^{2}}{M_{\mathrm{P}}^{2}} \frac{\hat{\sigma}^{\prime}}{\sigma^{\prime}}(1+\alpha \epsilon) g_{\mu \nu} .
\end{aligned}
$$

We can identify a number of effects. First, dressing of the $\kappa^{2}$ coupling (proportional to $\alpha$ ) can be absorbed into a redefinition of the Planck scale. It does not cause detuning of the sequester. By comparison, we cannot simply absorb (25b) into a redefinition of $\Lambda$ because of its $\kappa$ dependence.

Second, dressing of the $\Lambda$ coupling (proportional to $\beta$ ) is again responsible for breaking complete cancellation of the low-energy cosmological contribution between $T_{\mu \nu}$ and $\left\langle\left\langle T_{\mu}^{\mu}\right\rangle\right\rangle / 4$. The residual cosmological constant will be of order $\epsilon \Lambda_{\mathrm{IR}} \sim \epsilon M_{\mathrm{SM}}^{4}$ and therefore of a similar size to the estimates for the Einstein frame given at the end of Sec. II C. For numerical values we refer to the discussion given there.

Third, the $\Lambda$ dressing also causes inexact cancellation of the ultraviolet part $\Lambda_{\mathrm{UV}}$. The leftover piece has exactly the same structure as the left-over low energy loop contribution in $T_{\mu \nu}$, again because there is no distinction between these terms at the level of the Einstein equation. In the remainder of this paper we shall drop explicit dependence on $\Lambda_{\mathrm{UV}}$ and include its contribution in $\mathcal{L}_{\mathrm{m}}$ if required. Finally, we clearly see the contribution of the right-hand diagram in Fig. 2; this produces the term proportional to $\gamma M_{\mathrm{SM}}^{6} / \kappa^{2}[13]$.

\section{Sequestering the graviton loops}

Kaloper et al. observed that the troublesome $\gamma$ term appears in Eq. (27) as a consequence of its appearance in the $\kappa$ field equation [13]. If it could be removed from this field equation then terms of any order in $\kappa^{-2}$ contained in $T_{\mu \nu}$ would be sequestered as part of the usual cancellation between $T_{\mu \nu}$ and $\left\langle\left\langle T_{\mu}^{\mu}\right\rangle\right\rangle / 4$, at least in the absence of renormalizations to the $\Lambda$ coupling to the volume of spacetime.

In turn, graviton-loop contributions to the low-energy cosmological term contribute to the $\kappa$ field equation only because the graviton propagator carries a normalization of $\kappa^{-2}$. To decouple these contributions Kaloper et al. proposed the following formulation (which they described as "omnia sequestra") [13,30]

$$
\begin{aligned}
S= & \int \mathrm{d}^{4} x \sqrt{-g}\left(\frac{M_{\mathrm{P}}^{2}}{2} R+\theta R_{\mathrm{GB}}-\Lambda-\mathcal{L}_{\mathrm{m}}\right) \\
& +\sigma\left(\frac{\Lambda}{\mu^{4}}\right)+\hat{\sigma}(\theta) .
\end{aligned}
$$

Recall that we are now absorbing $\Lambda_{\mathrm{UV}}$, if present, into $\mathcal{L}_{\mathrm{m}}$. The normalization of the Einstein term reverts to the fixed Planck scale $M_{\mathrm{P}}$. Meanwhile we introduce the GaussBonnet density $R_{\mathrm{GB}}$ coupled to a rigid scalar $\theta$ that replaces $\kappa$. The Gauss-Bonnet density is defined by

$$
R_{\mathrm{GB}} \equiv R^{2}-4 R^{\mu \nu} R_{\mu \nu}+R^{\mu \nu \rho \sigma} R_{\mu \nu \rho \sigma} .
$$

In four dimensions its integral is proportional to a topological invariant, the Euler characteristic $\chi(M)$ of the manifold $M$. Because it is topological (it integrates to a boundary term), it follows that $R_{\mathrm{GB}}$ does not modify the form of the graviton propagator or its self-interaction vertices. The conclusion is that each internal graviton line scales like $M_{\mathrm{P}}^{-2}$ and carries no $\theta$ dependence. Operators in the quantum effective action that are built from diagrams containing such lines do not perturb the field equation for $\theta$.

Further, because of its topological character, the coefficient of the Gauss-Bonnet density is not renormalized. At the level of Feynman diagrams this follows because $\theta$ does not contribute to graviton vertices. Therefore there is no analogue of the diagrams in Fig. 1 for $R_{\mathrm{GB}}$. For the same reason, quantum corrections do not introduce $\theta$ dependence in $\mathcal{L}_{\mathrm{m}}$ at any order in the loop expansion. 
The coupling of $\Lambda$ to the spacetime volume will still be dressed by graviton loops, yielding Eq. (25b) with the replacement $\kappa^{2} \rightarrow M_{\mathrm{P}}^{2}$ in $\epsilon$. However, unlike Eqs. (25b) and (26), there is now no obstruction to absorbing the loop correction into a redefinition of $\Lambda$. Accordingly we do not expect detuning of the sequester in this case.

To verify this expectation consider the field equations following from (28) with the leading loop correction to the $\Lambda$ coupling included,

$$
\begin{gathered}
M_{\mathrm{P}}^{2} G_{\mu \nu}=T_{\mu \nu}-(1+\alpha \epsilon) \Lambda g_{\mu \nu}, \\
\frac{\sigma^{\prime}}{\mu^{4}}=(1+\alpha \epsilon) \int \mathrm{d}^{4} x \sqrt{-g} \\
\hat{\sigma}^{\prime}=-\int \mathrm{d}^{4} x \sqrt{-g} R_{\mathrm{GB}}
\end{gathered}
$$

From Eqs. (30b)-(30c) we conclude

$$
\frac{\hat{\sigma}^{\prime}}{\sigma^{\prime}} \mu^{4}=-(1-\alpha \epsilon)\left\langle\left\langle R_{\mathrm{GB}}\right\rangle\right\rangle .
$$

Because the Gauss-Bonnet density integrates to the Euler characteristic, up to a numerical factor, this is a relatively stringent condition on $\mu$. Assuming the derivatives $\sigma^{\prime}$ and $\hat{\sigma}^{\prime}$ are order unity ${ }^{8}$ it roughly requires $\mu \sim H_{\text {age }}$, where $H_{\text {age }}$ is the quantity defined in (14). See also Ref. [30].

Meanwhile, the trace of the Einstein equations requires

$$
R=\frac{4}{M_{\mathrm{P}}^{2}}(1+\alpha \epsilon) \Lambda-\frac{1}{M_{\mathrm{P}}^{2}} T^{\mu}{ }_{\mu} .
$$

As in the analyses given above, taking the spacetime expectation of this formula gives an expression for $\Lambda$ in terms of $\langle\langle R\rangle\rangle$ and $\left\langle\left\langle T^{\mu}{ }_{\mu}\right\rangle\right\rangle$. This expression should be used to eliminate $\Lambda$ from the Einstein equation. Finally, expressing $\left\langle\langle R\rangle\right.$ in terms of $\left\langle\left\langle R_{\mathrm{GB}}\right\rangle\right\rangle$ yields

$$
M_{\mathrm{P}}^{2} G_{\mu \nu}=T_{\mu \nu}-\frac{1}{4}\left\langle\left\langle T_{\rho}^{\rho}\right\rangle\right\rangle g_{\mu \nu}-L g_{\mu \nu},
$$

where $\ell$ is defined by (cf. Eqs. (11)-(12) of Ref. [13])

$$
\begin{aligned}
L^{2}= & \frac{3}{8} M_{\mathrm{P}}^{4}\left(\left\langle\left\langle R_{\mathrm{GB}}\right\rangle\right\rangle-\left\langle\left\langle W^{2}\right\rangle\right\rangle+\frac{2}{M_{\mathrm{P}}^{2}}\left\langle\left(T_{\mu \nu}-T g_{\mu \nu} / 4\right)^{2}\right\rangle\right\rangle \\
& \left.\left.-\frac{1}{6 M_{\mathrm{P}}^{4}}\left[\left\langle T^{2}\right\rangle\right\rangle-\langle\langle T\rangle\rangle^{2}\right]\right),
\end{aligned}
$$

where $T=T^{\rho}{ }_{\rho}$ and $W_{\mu \nu \rho \sigma}$ is the Weyl tensor derived from $g_{\mu \nu}$. This is exactly the result derived in Ref. [13]. As

\footnotetext{
${ }^{8}$ In our presentation, we are absorbing the integrated fluxes $\int F_{4}, \int \hat{F}_{4}$ into the definition of $\sigma, \hat{\sigma}$. If these factors are large they may modify conclusions based on dimensional analysis of (31).
}

expected, dressing of the $\Lambda$ coupling has no effect at the level of the effective Einstein equation. We conclude that extensive renormalizations of the coupling between $\Lambda$ and the spacetime volume do not de-tune sequestration in the formulation (28).

\section{CONCLUSIONS}

In this paper we have studied a class of radiative corrections to the sequester model proposed by Kaloper, Padilla and collaborators. Although the corrections we compute have previously been recognized, their effect has not been studied explicitly. The class of diagrams we study renormalize the couplings between the "rigid" scalar fields that are characteristic of the sequester scenario, and infrared properties of the spacetime such as its volume and integrated curvature.

In both the Einstein and Jordan frame formulations (given by Eqs. (1) and (19) in our notation), we find that these renormalizations disrupt complete sequestration of low-energy loop contributions. If the sequestered sector is the Standard Model, we find that these corrections very nearly produce an unacceptable cosmological term in excess of the observed value $\Lambda \sim\left(10^{-3} \mathrm{eV}\right)^{4}$. Whether or not a particular realization of the scenario yields an acceptable phenomenology then depends on how the global function $\sigma$ is engineered (and likewise for $\hat{\sigma}$ in the Jordanframe formulation).

Alternatively, if the sequestered sector contains higher mass particles such as supersymmetric partners with masses in excess of $10 \mathrm{TeV}$, the residual cosmological term is likely to be fatal. The situation could possibly be saved if physical scales are significantly rescaled in the effective Einstein frame metric. This is easiest to see in the explicit Einstein-frame description, where masses are rescaled by the conformal factor $\lambda$. We can possibly arrange for this rescaling $\lambda$ to be small, but such scenarios encounter other difficulties [11].

The simpler formulations of the sequester (those that do not invoke the Gauss-Bonnet density) are already known to "fail" in the sense that they do not capture contributions to the vacuum energy from diagrams that contain virtual gravitons. Although the renormalizations we have computed are related to these known failure modes, they are not the same. In most models the loop terms we compute are likely to be somewhat smaller, since the leading contribution involves two virtual gravitons and therefore scale as $\left(M_{\mathrm{SM}} / M_{\mathrm{P}}\right)^{4}$. This should be compared to a single-graviton loop scaling as $\left(M_{\mathrm{SM}} / M_{\mathrm{P}}\right)^{2}$ as in the left-hand diagram of Fig. 2.

We find that these renormalizations do not affect the most developed formulation of the sequester, given by Eq. (28) in our notation. In this formulation, dressing of the $\Lambda$ interaction can be absorbed into a redefinition of $\Lambda$ itself and is therefore harmless. 
Whether or not one finds the sequester a plausible solution to the naturalness problem of the cosmological constant depends on whether we are prepared to accept its key ingredient- the introduction of nongravitating sectors in the action that are shielded from gravity: they do not source gravitational fields, and they do not interact with gravitons. For related models utilising a similar premise see Refs. [24,26,31,32]. This is the cost of entry for all versions of the sequester scenario. Once accepted, it is only necessary to arrange for the large low-energy loop contribution to be "stored" in these non-gravitating sectors.

At the level of the effective actions used in this paper there is little more that can be said. In particular, we have not been able to apply "naturalness" arguments to the nongravitating functions $\sigma$ and $\hat{\sigma}$, because to do so would require specification of a microscopic theory that describes the fluxes $F_{4}, \hat{F}_{4}$ that project out local degrees of freedom from the rigid fields $\Lambda, \kappa$ and $\theta$ (depending on the formulation in use). These nongravitating sectors are the final repository for sequestered vacuum energy. If it is possible to build models in which these sectors have their own microscopic description, it would be very interesting to apply naturalness criteria to the model as a whole.

\section{ACKNOWLEDGMENTS}

M. G. L. acknowledges support from the UK Science and Technology Facilities Council via Research Training Grant No. ST/M503836/1. D. S. acknowledges support from the Science and Technology Facilities Council [grant number ST/L000652/1]. We would like to thank Chris Byrnes for helpful discussions throughout this project. We thank Chris Byrnes and Tony Padilla for helpful comments on an early version of this paper.
[1] F. Wilczek, in The Very Early Universe (1983), pp. 9-28.

[2] S. Weinberg, Rev. Mod. Phys. 61, 1 (1989).

[3] S. Rugh and H. Zinkernagel, Stud. Hist. Phil. Sci. B 33, 663 (2002).

[4] N. Straumann, in 18th IAP Colloquium on the Nature of Dark Energy: Observational and Theoretical Results on the Accelerating Universe (2002).

[5] C. P. Burgess and D. London, Phys. Rev. D 48, 4337 (1993).

[6] D. H. Lyth, Prog. Theor. Phys. Suppl. 190, 107 (2011).

[7] M. S. Sloth, Int. J. Mod. Phys. D 19, 2259 (2010).

[8] M. Maggiore, Phys. Rev. D 83, 063514 (2011).

[9] G. Mangano, Phys. Rev. D 82, 043519 (2010).

[10] N. Kaloper and A. Padilla, Phys. Rev. Lett. 112, 091304 (2014).

[11] N. Kaloper and A. Padilla, Phys. Rev. D 90, 084023 (2014); 90, 109901(A) (2014).

[12] N. Kaloper, A. Padilla, D. Stefanyszyn, and G. Zahariade, Phys. Rev. Lett. 116, 051302 (2016).

[13] N. Kaloper and A. Padilla, Phys. Rev. Lett. 118, 061303 (2017).

[14] B. Richter, Phys. Today 59, 10, 8 (2006).

[15] S. Hossenfelder, Lost in Math: How Beauty Leads Physics Astray (Basic Books, New York, 2018).

[16] G.F. Giudice, Proc. Sci., EPS-HEP2013 (2013) 163 [arXiv:1307.7879].

[17] P. Williams, Stud. Hist. Phil. Sci. B 51, 82 (2015).
[18] G.F. Giudice, The dawn of the post-Naturalness era, in From My Vast Repertoire...: Guido Altarelli's Legacy, edited by A. Levy, S. Forte, and G. Ridolfi (World Scientific, Singapore, 2019), pp. 267-292.

[19] G. 't Hooft, NATO Sci. Ser. B 59, 135 (1980).

[20] A. Padilla, arXiv:1502.05296.

[21] S. W. Hawking, General Relativity, edited by S.W. Hawking and W. Israel (Cambridge University Press, Cambridge, England, 1979).

[22] M. Tanabashi et al. (Particle Data Group), Phys. Rev. D 98, 030001 (2018).

[23] A. Padilla, J. High Energy Phys. 01 (2019) 175.

[24] L. Bordin, F. Cunillera, A. Lehebel, and A. Padilla, Phys. Rev. D 101, 085012 (2020).

[25] B. K. El-Menoufi, S. Nagy, F. Niedermann, and A. Padilla, Classical Quantum Gravity 36, 215014 (2019).

[26] D. Sobral-Blanco and L. Lombriser, Phys. Rev. D 102, 043506 (2020).

[27] S. Alexander, G. Herczeg, J. Liu, and E. McDonough, Phys. Rev. D 102, 083526 (2020).

[28] I. Oda, arXiv:1709.08189.

[29] P. Brax, C. Burrage, A.-C. Davis, D. Seery, and A. Weltman, Phys. Lett. B 699, 5 (2011).

[30] B. Coltman, Y. Li, and A. Padilla, J. Cosmol. Astropart. Phys. 06 (2019) 017.

[31] I. Oda, Phys. Rev. D 95, 104020 (2017).

[32] S. M. Carroll and G. N. Remmen, Phys. Rev. D 95, 123504 (2017). 